\title{
BMJ Open Nature of adverse events with opioids in hospitalised patients: a post-hoc analysis of three patient record review studies
}

\author{
Bernadette Clara Francisca Maria Schutijser (10 , ${ }^{1}$ Irene Jongerden, ${ }^{1}$ \\ Joanna Ewa Klopotowska (D) ,2 Marco Moesker (D) , ${ }^{1}$ Maaike Langelaan, ${ }^{3}$ \\ Cordula Wagner, ${ }^{1,3}$ Martine de Bruijne (i) ${ }^{1}$
}

To cite: Schutijser BCFM, Jongerden I, Klopotowska JE, et al. Nature of adverse events with opioids in hospitalised patients: a post-hoc analysis of three patient record review studies. BMJ Open 2020;10:e038037. doi:10.1136/ bmjopen-2020-038037

- Prepublication history and additional material for this paper are available online. To view these files, please visit the journal online (http://dx.doi. org/10.1136/bmjopen-2020038037).

Received 26 February 2020 Revised 13 August 2020 Accepted 18 August 2020

A) Check for updates

(c) Author(s) (or their employer(s)) 2020. Re-use permitted under CC BY-NC. No commercial re-use. See rights and permissions. Published by BMJ.

${ }^{1}$ Public and Occupational Health, Amsterdam UMC Locatie VUmc, Amsterdam, The Netherlands ${ }^{2}$ Medical Informatics, Amsterdam UMC Locatie AMC, Amsterdam, The Netherlands ${ }^{3}$ NIVEL, Utrecht, The Netherlands

Correspondence to Bernadette Clara Francisca Maria Schutijser;

b.schutijser@amsterdamumc.nl

\section{ABSTRACT}

Objective Opioids are increasingly prescribed and frequently involved in adverse drug events (ADEs). The underlying nature of opioid-related ADEs (ORADEs) is however understudied. This hampers our understanding of risks related to opioid use during hospitalisation and when designing interventions. Therefore, we provided a description of the nature of ORADEs.

Design A post-hoc analysis of data collected during three retrospective patient record review studies (in 2008, 2011/2012 and 2015/2016).

Setting The three record review studies were conducted in 32 Dutch hospitals.

Participants A total of 10917 patient records were assessed by trained nurses and physicians.

Outcome measures Per identified ORADE, we described preventability, type of medication error, attributable factors and type of opioids involved. Moreover, the characteristics of preventable and non-preventable ORADEs were compared to identify risk factors.

Results Out of 10917 patient records, 357 ADEs were identified, of which $28(8 \%)$ involved opioids. Eleven ORADEs were assessed as preventable. Of these, 10 were caused by dosing errors and 4 probably contributed to patients' death. Attributable factors identified were mainly on patient and organisational levels. Morphine and oxycodone were the most frequently involved opioids. The risk for ORADEs was higher in elderly patients.

Conclusions 0 nly $8 \%$ of ADEs identified in our sample were related to opioids. Although the frequency is low, the risk of serious consequences is high. We recommend to use our findings to increase awareness among physicians and nurses. Future interventions should focus on safe dosing of opioids when prescribing and administering, especially in elderly patients.

\section{INTRODUCTION}

Over the past decades, prescription of opioids has substantially increased worldwide. ${ }^{12}$ Moreover, the rise in addiction rates and deaths resulting from opioid overdoses has urged physicians to call out an opioid crisis. ${ }^{3}$ In the Netherlands, the prescription of
Strengths and limitations of this study

- This study was based on data gathered during three national retrospective patient record review studies conducted in 2008, 2011/2012 and 2015/2016 within 32 Dutch hospitals.

- During all three studies, a broad and randomly selected sample of all hospital admissions of patients were reviewed to assess the nature and preventability of adverse drug events with opioids.

- Our study population was stratified, resulting in an over-representation of in-hospital deceased patients.

- The low frequency of opioid-related adverse drug events limited a comparison of events over time among the three study periods.

oxycodone has increased almost fivefold over 10 years (from 96000 users in 2008 to 485000 users in 2018). ${ }^{4}$ This increase may however not only lead to more addiction but may also affect the number of opioid-related adverse drug events (ADEs) in hospitals.

Opioids are frequently involved in $\mathrm{ADEs}^{5-7}$ and approximately in 2\%-14\% of all patients. ${ }^{8-12}$ ADEs are unintended injuries from a medical intervention related to drugs. ${ }^{13}$ Opioid-related ADEs (ORADEs) occur frequently, specifically in paediatric, ${ }^{714}$ palliative $^{15}$ and surgical patients. ${ }^{101116}$ ORADEs are often caused by errors such as omissions or incorrect dosing. ${ }^{7141517}$ In addition, approximately $11 \%$ of ORADEs among hospitalised patients cause severe or even fatal patient harm, ${ }^{18}$ and also due to the fast therapeutic effects of opioids. Besides these severe consequences, ORADEs lead to significantly higher healthcare costs. ${ }^{9} 1016$

Our current knowledge about the incidence of ORADEs and their underlying nature is mostly based on medication-related incident 
reports. ${ }^{7} 141517$ However, a comprehensive patient chart review provides the most reliable information on ADEs in hospitals, while incident reports suffer from severe under-reporting. ${ }^{19}{ }^{20}$ Furthermore, ORADE studies based on incident reports were usually conducted at one point in time or within one hospital or at a specific department. 7141517 The few ORADE studies based on comprehensive patient chart review were mainly conducted within a surgical population. ${ }^{101116}$

Therefore, and also motivated by the opioid crisis, we have conducted an indepth analysis of ORADEs using data gathered during three consecutive national adverse event (AE) studies in the Netherlands in which patient record review was applied. To our knowledge, no such longitudinal multicentre study on ORADEs in a diverse inpatient population and using a comprehensive ADE detection method has been published. The aim of this study was to provide a detailed description of the underlying nature of ORADEs. By doing so, we hope to increase awareness and provide recommendations on how to prevent ORADEs in future hospitalised patients.

\section{METHODS}

\section{Design and setting}

We conducted a post-hoc analysis of data that were collected during three national retrospective patient record review studies conducted in 2008, 2011/2012 and 2015/2016. The aim of these studies was to identify AEs and ADEs in Dutch hospitals. A detailed description of the methodology used in these studies was previously published and comparable with other international AE studies. $^{21}{ }^{22}$ In summary, for the 2008 and 2011/2012 studies, a random sample of 20 hospitals participated. In 2015/2016, a new random sample of 19 hospitals were selected, of which 7 had previously participated in two of the earlier studies. Both samples were stratified for hospital type and representation of urban and rural areas. In 2008 and 2011/2012, 200 patient records per hospital were randomly selected for review: 100 records of discharged patients and 100 records of in-hospital deceased patients. The 2015/2016 study was limited to 150 in-hospital deceased patients per hospital because the frequency of preventable AEs remained unchanged for in-hospital deceased patients in both the 2008 and the $2011 / 2012$ measurements. ${ }^{23-25}$ Records of patients younger than 1 year and of patients admitted at the departments of psychiatry and obstetrics were excluded because other expertise is necessary to detect AEs in these patients. The random selection of patient records was conducted by the participating hospitals with clear instructions of the researchers.

\section{Review procedure: AE studies}

During all three AE studies, selected patient records were reviewed for the occurrence of AEs, including ADEs. In figure 1, a schematic overview of the review process in the national studies and this study is presented. In summary, the review process consisted of two phases. In phase 1, the records were screened for potential AEs by trained independent nurses. When predefined triggers were found, indicating an AE might have occurred, the record was labelled for an indepth review by a trained independent physician. Independent means that the physicians and nurses never had an employment contract in the participating hospitals. The physicians were highly experienced and specialised in surgery, internal medicine or neurology, and during the record review studies they had access to all information on the electronic patient records. Besides, $10 \%$ of all patient records were reviewed by two physicians to determine inter-rater reliability. Validity of this scoring system has not been tested, but it has been used widely in $\mathrm{AE}$ studies for over 20 years and the ratings of the system did not change in that time..$^{21-2326-29}$ Prior to the study, both nurses and physicians had training sessions in which cases were discussed to enhance the quality and standardisation of the review process.

An AE was defined by three criteria: (1) an unintended physical or mental injury; (2) the injury resulted in prolongation of hospital stay, temporary or permanent disability, or death; and (3) the injury was caused by healthcare management rather than the patient's underlying disease. ${ }^{232728}$ An AE was scored as caused by the healthcare (causality) if the likelihood score was equal to or greater than 4 based on a 6-point Likert scale, with (virtually) no evidence (1), slight to modest evidence (2), not likely but borderline (3), more likely but borderline (4), moderate to strong evidence (5), or (virtually) certain evidence (6) of management causation. The scoring system was used in all three record review studies and the physicians made the judgements about causality and preventability based on all the available information of the patient's condition and taking into account the guidelines.

If an $\mathrm{AE}$ was identified, the independent physicians (hereafter: experts) assessed each AE on cause (diagnostic, surgery, non-invasive procedure, medication, other clinical activities, admission and other), preventability, possible contribution to death and attributable factors. The attributable factors were based on the taxonomy of the Eindhoven Classification Model and consisted of the main categories: technical, care, organisational, patient-related, violation and other. ${ }^{30}$ An AE was considered to be preventable when the care given fell below the current level of expected performance of practitioners or systems. Before the physicians answered the question about preventability, they were required to respond to 13 questions to add more structure to the review process (see online supplemental table 1), for example, if there was a complex medical history, if the patient had comorbidity and whether another physician would repeat this treatment. Preventability was also assessed on a 6-point Likert scale, with almost no evidence (1), slight to modest evidence (2), modest evidence but borderline (3), modest to strong evidence (4), strong evidence (5), or almost certain evidence (6) of preventability. A score 


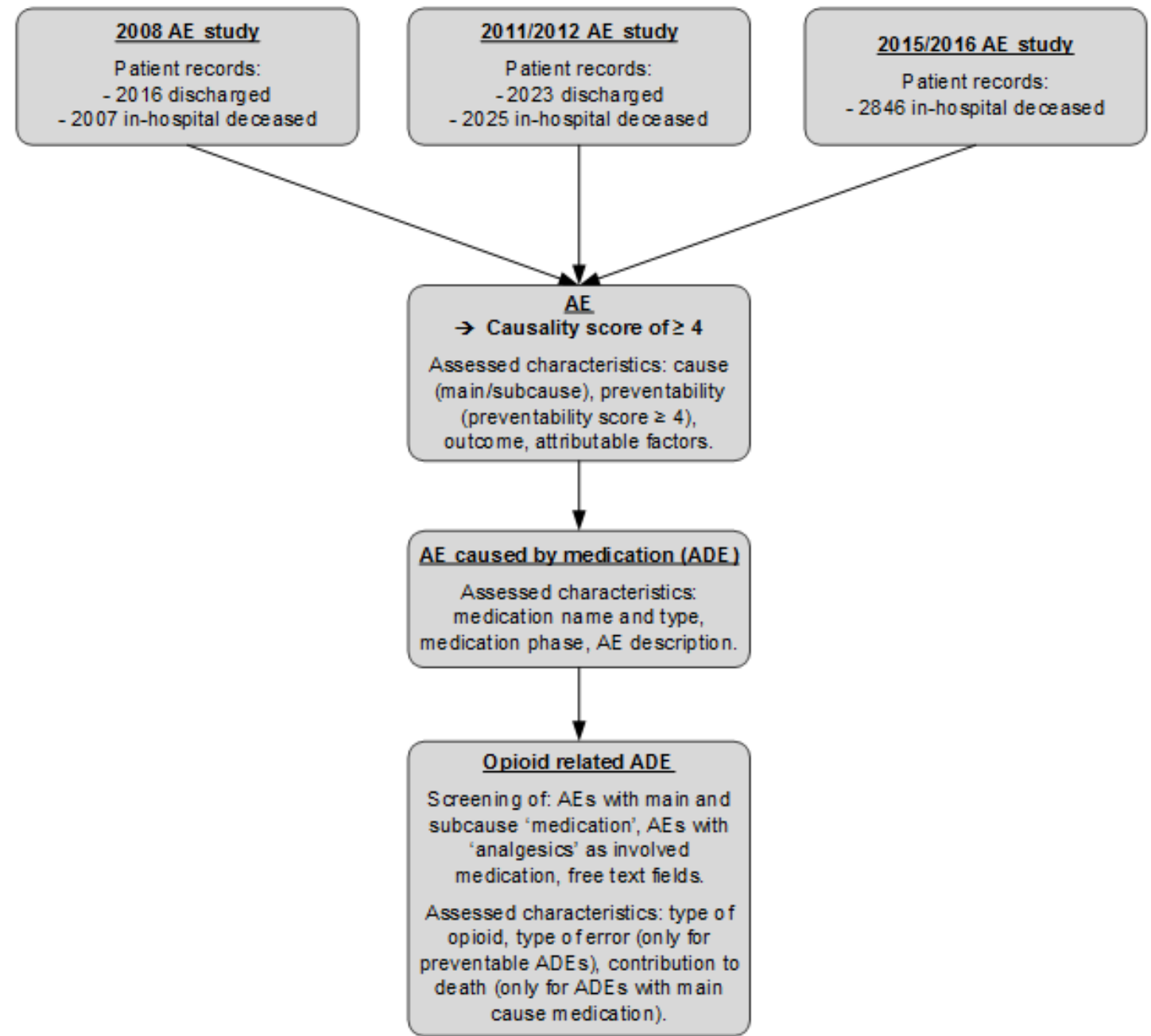

Figure 1 Overview of the three Dutch adverse event studies and our study. ADE, adverse drug event; AE, adverse event.

of 4-6 indicated that the reviewer assessed the $\mathrm{AE}$ as having a greater than $50 \%$ chance of being potentially preventable.

Furthermore, for each patient the following characteristics were registered: gender, age, length of hospital stay, urgency of admission, whether patients were terminally ill prior to the admission, the number of involved medical specialists, department of admission, type of procedure and comorbidity. The latter was divided into no, minor, moderate and severe comorbidity, and was assessed by the experts after careful review of the information on the patient record. Also, one organisational characteristic (type of hospital: university, tertiary teaching or general) and one $\mathrm{AE}$ characteristic (weekend or holiday at the time of the $\mathrm{AE}$ ) were registered.

When an AE was medication-related (ADE), the following additional characteristics were registered by the experts: name and type of medication involved, medication phase, a description of the ADE and whether the ADE possibly contributed to the patient's death. The medication phases were classified into ordering, transcribing, dispensing, administering and monitoring. ${ }^{3132}$ The possible contribution to the patient's death was only registered for ORADEs, with 'medication' as a main cause of the event and not for ADEs with 'medication' as a subcause.

All data were entered into a national $\mathrm{AE}$ database specifically designed for these AE studies.

\section{Review procedure: ORADEs}

For our study, we used the national AE database to identify ORADEs (figure 1). One researcher (BCFMS) conducted the screening of the database and retrieved several preselected variables: (1) AEs with the main classification cause 'medication' as well as AEs with 'medication' as a subcause; and (2) AEs with 'analgesics' as involved medication. Furthermore, two free-text fields were selected: the summary of the AEs and the preventability assessment. A second researcher (MM) independently doublechecked the selection procedure.

All identified ORADEs were then classified by BCFMS on the type of opioids involved using the WHO Anatomical Therapeutic Chemical (WHO ATC) classification. ${ }^{33}$ For the preventable ORADEs, the type of medication error was classified according to a data-driven analysis of the free-text summaries of the ADEs. The classification of ORADEs was double-checked by two senior researchers 
(JEK and $\mathrm{IJ}$ ) and any discrepancies were resolved by consensus.

\section{Outcomes}

To provide insight into the nature of the ORADEs, each ORADE case was summarised by gender, age of the patient (categorised in steps of 10 years for privacy reasons), type of opioid involved, attributable factors and preventability. When the ORADE was preventable, then the type of medication error and medication phase were also described. Furthermore, in order to identify risk factors, we compared the outcome variables between preventable and non-preventable ORADEs.

\section{Data analysis}

Only descriptive statistics were used in this study. Descriptives are presented as median (age and length of hospital stay) or frequency (gender, comorbidity, type of opioids and attributable factors and so on). Patient and hospital characteristics are presented on the patient level and ORADE characteristics are presented on the AE level. Inter-rater reliability among nurses and physicians was addressed in terms of positive and negative agreement frequencies. ${ }^{34}$ All analyses were conducted using STATA V.14.1 and double-checked by a second researcher (MM) and a statistician (PS).

\section{Patient and public involvement}

Patients or the public were not involved in the design, or conduct, or reporting, or dissemination plans of our research.

\section{RESULTS}

In total, 10917 records were screened during the three $\mathrm{AE}$ studies. The patient records of discharged and deceased patients were equally distributed among male and female patients. Most patients were hospitalised for a non-elective procedure (table 1). In 1150 patient records, at least one AE was detected, with a total of 1240 AEs. When detecting the predefined triggers, positive agreement between nurses varied between $76.0 \%$ and $91.5 \%$. When detecting AEs, positive agreement between physicians varied between $53.4 \%$ and $63.3 \%$. For assessing preventability, positive agreement between physicians varied between $71.4 \%$ and $73.3 \%$. Overall, agreement frequencies were moderate. More detailed information about the inter-rater reliability is presented in online supplemental table 2.

\section{Opioid-related ADEs}

Of 1240 AEs, 357 (29\%) were medication-related (ADEs). In $28(8 \%)$ ADEs, opioids were involved. These ADEs are summarised in detail in table 2, and included 24 ADEs with 'medication' as a main cause and four ADEs with 'medication' as a subcause. The ORADEs occurred in 27 patients; one patient experienced two ORADEs. Most patients with ORADEs involved female patients (59\%). The median age of the patients was 76 years (IQR: 66-83), and the median length of hospital stay was 7 days (IQR: 4-16). Most patients had moderate to significant comorbidity $(70 \%)$ and had three medical specialists during the admission (78\%) (table 3).

\section{Nature of ORADEs: preventability}

According to the experts, 11 (39\%) out of the 28 ORADEs were considered as potentially preventable (table 4). Non-preventable (31\%) ORADEs occurred slightly more during weekends and holidays than preventable ADEs $(18 \%)$. Moreover, most preventable and non-preventable ORADEs occurred during day shifts (08:00-17:00).

\section{Nature of ORADEs: medication errors and phase}

Of the 11 potentially preventable ORADEs, 10 (91\%) were caused by dosing errors, of which 6 were during the prescribing phase (cases 1, 3, 7, 8, 9 and 10) and 4 during the administration phase (cases 2, 4, 5 and 6) (table 2). Of the 10 dosing errors, 6 occurred in elderly patients $(\geq 70$ years) (cases 1, 3, 4, 5, 8 and 9) and 2 around patients' discharge (cases 2 and 7). The remaining one preventable ORADE (case 11) was related to incorrect decision making. Finally, the experts assessed the consequences of the ORADEs (multiple options possible). In eight ORADEs, an intervention or extra treatment was needed, in two ORADEs the patients had a prolonged hospital stay, and four preventable ORADEs possibly contributed to the death of the patient (cases 5, 6, 8 and 9).

\section{Nature of ORADEs: attributable factors}

The attributable factors involved in ORADEs were carerelated (knowledge, skills, monitoring, verification and coordination of care) and patient-related (comorbidity, age, a demanding patient or a patient with an intellectual disability) (table 4). Of preventable ORADEs, eight were care-related and six were patient-related. For nonpreventable ORADEs, 3 were care-related and 10 were patient-related. However, in three of the cases of nonpreventable ORADEs, the attributable factors could not be assessed by the experts due to insufficient information on the patient records.

\section{Nature of ORADEs: medications involved}

Out of the 11 preventable ADEs, 8 occurred with opioids with ATC code N02AA, which are morphine and oxycodone (table 4). Non-preventable ORADEs occurred with opioids mainly with ATC code N02AA (morphine and oxycodone, $53 \%$ ).

\section{DISCUSSION}

In three national patient record studies with 4-year intervals, we found 28 ADEs caused by opioids. These ADEs correspond with $8 \%$ of all identified ADEs and $0.3 \%$ of all studied patient records. Of the 28 ORADEs, 11 (39\%) were assessed as potentially preventable, involving mostly morphine and oxycodone. Dosing errors during the prescription and administration phase were the most common cause of preventable ORADEs and occurred 
Table 1 Patient and hospital characteristics of all reviewed patient records, including adverse events per study period and discharge status

\begin{tabular}{|c|c|c|c|c|c|}
\hline & \multicolumn{5}{|c|}{ Study period and discharge status } \\
\hline & \multicolumn{2}{|c|}{2008} & \multicolumn{2}{|l|}{$2011 / 2012$} & \multirow{2}{*}{$\begin{array}{l}\text { 2015/2016 } \\
\text { Deceased }\end{array}$} \\
\hline & Discharged & Deceased & Discharged & Deceased & \\
\hline \multicolumn{6}{|l|}{ Hospital characteristics* } \\
\hline Patient records, $\mathrm{n}$ & 2016 & 2007 & 2023 & 2025 & 2846 \\
\hline General hospital records, $\mathrm{n}(\%)$ & $1013(50)$ & $1015(51)$ & $794(39)$ & $813(40)$ & $1197(42)$ \\
\hline Tertiary teaching hospital records, n (\%) & $608(30)$ & $593(30)$ & $822(41)$ & $820(40)$ & $1052(37)$ \\
\hline Academic hospital records, $\mathrm{n}(\%)$ & $395(20)$ & $399(20)$ & $407(20)$ & $392(19)$ & $597(21)$ \\
\hline \multicolumn{6}{|l|}{ Patient characteristics* } \\
\hline Male sex, n (\%) & $999(50)$ & $1067(53)$ & $1027(51)$ & $1062(52)$ & $1524(54)$ \\
\hline Age (years), median (IQR) & $62(47-75)$ & $77(67-84)$ & $63(48-75)$ & $77(68-84)$ & $77(68-85)$ \\
\hline Length of stay (days), median (IQR) & $4(2-8)$ & $7(3-14)$ & $3(2-7)$ & $6(2-13)$ & $4(1-11)$ \\
\hline Non-elective admission, n (\%) & $1038(51)$ & $1708(85)$ & $1063(53)$ & $1775(88)$ & $2496(88)$ \\
\hline \multicolumn{6}{|l|}{ Admission department, $\mathrm{n}(\%)$} \\
\hline Surgery & $481(24)$ & $276(14)$ & $472(23)$ & $239(12)$ & $340(12)$ \\
\hline Cardiology & $290(14)$ & $291(15)$ & $272(13)$ & $247(12)$ & $360(13)$ \\
\hline Internal medicine & $364(18)$ & $599(30)$ & $365(18)$ & $597(29)$ & $876(31)$ \\
\hline Orthopaedics & $226(11)$ & $33(2)$ & $225(11)$ & $26(1)$ & $29(1)$ \\
\hline Neurology & $150(7)$ & $219(11)$ & $133(7)$ & $193(10)$ & $269(9)$ \\
\hline Lung diseases & $117(6)$ & $259(13)$ & $126(6)$ & $300(15)$ & $347(12)$ \\
\hline Urology & $109(5)$ & $18(1)$ & $111(5)$ & $28(1)$ & $23(1)$ \\
\hline Other & $279(14)$ & $312(16)$ & $319(16)$ & $395(20)$ & $602(21)$ \\
\hline Underwent invasive procedure, $\mathrm{n}(\%)$ & $925(46)$ & $423(21)$ & $918(45)$ & $403(20)$ & $461(16)$ \\
\hline \multicolumn{6}{|l|}{ Adverse event occurrence†‡ } \\
\hline AE, n (\%) & $161(8)$ & $351(16)$ & $157(8)$ & $259(12)$ & $312(10)$ \\
\hline ADE, $n$ (\% within population) & $37(2)$ & $93(4)$ & $40(2)$ & $76(4)$ & $111(4)$ \\
\hline ADE, $n$ (\% within adverse event) & $37(23)$ & $93(27)$ & $40(25)$ & $76(29)$ & $111(36)$ \\
\hline ORADE, $\mathrm{n}$ (\% within population) & $1(0)$ & $7(0)$ & $2(0)$ & $8(0)$ & $10(0)$ \\
\hline ORADE, n (\% within ADEs) & $1(3)$ & $7(8)$ & $2(5)$ & $8(11)$ & $10(9)$ \\
\hline
\end{tabular}

${ }^{*}$ Presented on the patient record level.

†Presented on the AE level.

¥Total number of AEs: 1240; total number of ADEs: 357; total number of opioid-related ADEs: 28.

$A D E$, adverse drug event; $A E$, adverse event; ORADE, opioid-related adverse drug event.

most often in elderly patients. Four preventable ORADEs probably contributed to patients' death. Finally, attributable factors for ADEs were mostly care-related and patient-related.

In this study, the percentage of ORADEs of all patient records $(0.3 \%)$ was low, also in comparison with previously conducted ORADE studies that focused on large populations $(11 \%-14 \%) .{ }^{1011} 16$ However, two of these studies were based on large databases and all involved surgical patients who often receive opioids postoperatively. We focused on a broad hospitalised patient population, both surgical and non-surgical. Furthermore, the difference in ORADE occurrence might be explained by differences in the used ADE definition. For example, instead of using all ORADEs, that is, including side effects of opioids, in our study only ADEs that resulted in severe patient harm were included. This means that ADEs resulted in prolongation of hospital stay, temporary or permanent disability, or death. Furthermore, only ADEs with a causality likelihood score of equal or greater than 4 were included, which means that the experts indicated an ADE as having a greater than $50 \%$ chance of being caused by healthcare. Should we have selected the cases with causality likelihood scores of 1-3 as well, then we could determine at least 2500 additional cases on whether medication and opioids were related. However, we did not determine these 2500 cases, since we wanted to stay true to the definition of an $\mathrm{AE}$ (at least 4 on the 6-point Likert scale), and we did not consider it ethical to change the method of the study afterwards. 
Table 2 Descriptions of the 28 opioid-related adverse drug events divided into preventable and non-preventable

Preventability score (1-6)† and type of

Case Description* errorł

Preventable opioid-related ADEs

Cause: dosing errors

1 A man, 90-99 years, admitted with pain after a fall. Oxycodone for the pain was unintentionally prescribed twice instead of once and also administered twice (dose unknown). This resulted in drowsiness.

2 A man, 60-69 years, suffering from colon cancer and liver metastases, was admitted for optimising his analgesics medication. On returning from his weekend leave, he was diagnosed with oxycodone intoxication. During hospital stay, he received a too high dose of the opioid antagonist naloxone ( $1 \mathrm{mg}$ instead of the ordered $0.4 \mathrm{mg}$ ), which caused confusion and agitation.

3 A woman, 70-79 years, admitted with a pelvic fracture after a fall. A too high dose (dose unknown) of oxycodone was prescribed and administered resulting in hypotension and drowsiness. Consequently, she needed to be transferred to the intensive care unit. A woman, 80-89 years, admitted with malaise after a fall. During her admission she received a too high dose of morphine. In her patient record, the morphine was ordered as 'as needed' (PRN). In the medication list, the morphine was ordered ' 6 times a day' (dose unknown). This resulted in drowsiness.

5 A woman, 70-79 years, admitted for a plastic surgery. A high dose of intravenous administered anaesthetic/pain medication (dose and medication type unknown) caused hypoventilation and a myocardial infarct. The myocardial infarct was discovered too late. She was resuscitated and ventilated. Her death was possibly caused by a hospitalacquired pneumonia.

6 A woman, 50-59 years, admitted due to an aspiration pneumonia, was administered morphine. The pump mode was set at $13 \mathrm{~mL} /$ hour instead of $8 \mathrm{~mL} /$ hour as ordered. This possibly resulted in an epileptic insult requiring ventilation.

7 A man, 60-69 years, readmitted to the hospital due to a collapse at home. He was previously hospitalised for treatment 4 of rib fractures and COPD Gold IV. At discharge, the doses of fentanyl and oxycodone had been significantly increased (prescribing error) to $20 \mathrm{mg} \mathrm{4-6}$ times a day. Monitoring the effects of increasing these opioid doses was not conducted. A woman, 80-89 years, admitted with osteoporosis, received at home $5 \mathrm{mg}$ morphine two times per day for her back pain. The dosage was increased to subcutaneous $5 \mathrm{mg}$ four times a day during hospital stay. Three days later, a paralytic ileus was discovered. A lower morphine dose was more appropriate for this elderly woman.

9 A woman, 80-89 years, admitted with abdominal pain due to kidney bleeding. She received morphine injections daily, varying from 2 to 6 subcutaneous injections of $2.5 \mathrm{mg}$ per day along with transdermal fentanyl $12 \mu \mathrm{g}$ hourly. Severe hypercapnia eventually caused her death.

10 A boy, 0-9 years, with Down syndrome, was acutely ill due to a laryngitis. He was difficult to ventilate and received antibiotics and sedatives including opioids. He was transferred to another hospital following detubation. Here, his methadone intake was reduced resulting in a delirium (dose unknown). Initially he improved, but one day unexpectedly he was found dead. It is unclear why this patient received methadone, but reducing the methadone intake may have been the problem.

Cause: incorrect decision making

11 A woman, 60-69 years, admitted for a laminectomy. Postoperatively she developed an ileus caused by severe constipation aggravated by administered morphine. Macrogol oral suspension (dose unknown) instead of an enema was given as treatment, which was insufficient to resolve, and the ileus and colon perforation occurred. Untreatable abdominal septic complications followed.

Non-preventable opioid-related ADEs

12 A woman, 80-89 years, admitted due to total knee replacement. Postoperatively, drowsiness, hypotension and oliguria occurred, possibly caused by the epidural medication sufentanil (dose unknown). This may have led to a small (administration error) asymptomatic myocardial infarct.

13 A man, 80-89 years, admitted with a perforated stomach ulcer and known stomach cancer. His extreme, not previously 3 known, sensitivity to morphine postoperatively (dose unknown) resulted in recurrent apnoea.

14 A woman, 60-69 years, suffering from lung cancer, was admitted with severe back and limb pain related to bone metastases. She was treated with transdermal fentanyl $300 \mu \mathrm{g} /$ hour. This resulted in drowsiness and hypoventilation.

(other error)

2

(prescribing error)

15 A woman, 80-89 years, known with breast cancer and multiple lung metastases. She received tramadol (dose unknown) 2 for the pain, which have been stopped due to drowsiness.

16 A man, 70-79 years, admitted with severe heart failure. He received morphine $2.5 \mathrm{mg}$ for the pain. As a result of increased, not previously known, sensitivity to morphine, his saturation dropped. (unknown) (unknown)

2

(other error)

17 A man, 90-99 years, admitted due to stroke and a lot of pain. The nurse administered $10 \%$ of the prescribed dose (dose 2 unknown) of morphine on two occasions, which caused unnecessary suffering.

(administration error)

(unknown) COPD and hospital-acquired pneumonia after receiving morphine (dose unknown).

19 A woman, 60-69 years, admitted with a reoccurrence of drowsiness, hypoventilation and difficulties with waking up which was the result of a dose of $5 \mathrm{mg}$ of methadone being administered in the hospital.

(prescribing and administration error) 


\begin{tabular}{|c|c|c|}
\hline 20 & $\begin{array}{l}\text { A woman, } 60-69 \text { years, had a blood pressure drop following the administration of morphine (dose unknown) in the } \\
\text { recovery room. }\end{array}$ & $\begin{array}{l}1 \\
\text { (other error) }\end{array}$ \\
\hline 21 & $\begin{array}{l}\text { A woman, } 70-79 \text { years, admitted with pain related to severe Kahler disease. For the pain, she received opioids } \\
\text { (unknown which type and dose). The opioids caused drowsiness, and because of the drowsiness she choked once. This } \\
\text { caused pneumonia. The patient died during hospitalisation. }\end{array}$ & $\begin{array}{l}1 \\
\text { (other error) }\end{array}$ \\
\hline 22 & $\begin{array}{l}\text { A man, } 70-79 \text { years, received transdermal fentanyl and oxycodone } 5 \mathrm{mg} \text { daily up to six times due to metastases in the } \\
\text { hip. This caused apraxia and confusion. }\end{array}$ & $\begin{array}{l}1 \\
\text { (unknown) }\end{array}$ \\
\hline 23 & $\begin{array}{l}\text { A woman, } 80-89 \text { years, admitted for occlusion of an artery in her leg. She received a morphine infusion }(0.5-1.0 \mathrm{mg} / \mathrm{hour}) \\
\text { causing hypoventilation with a good response to naloxone. }\end{array}$ & $\begin{array}{l}1 \\
\text { (administration error) }\end{array}$ \\
\hline 24 & $\begin{array}{l}\text { A man, } 80-89 \text { years, admitted due to obstructive laryngeal cancer, was prescribed anticoagulants. This resulted in } \\
\text { haematoma, along with severe abdominal pain for which he received morphine (dose unknown), after which he died. }\end{array}$ & $\begin{array}{l}1 \\
\text { (other error) }\end{array}$ \\
\hline 25 & $\begin{array}{l}\text { A man, } 60-69 \text { years, admitted with an acute respiratory insufficiency due to pneumonia. He received methadone } 20 \mathrm{mg} \\
\text { two times per day, causing hypoventilation on two occasions. This needed to be treated with naloxone. }\end{array}$ & $\begin{array}{l}1 \\
\text { (prescribing error) }\end{array}$ \\
\hline 26 & $\begin{array}{l}\text { A woman, } 80-89 \text { years, suffered from pain due to rib fractures caused by resuscitation. She received sufentanil (dose } \\
\text { unknown), which led to bronchospasm. }\end{array}$ & $\begin{array}{l}1 \\
\text { (unknown) }\end{array}$ \\
\hline 27 & $\begin{array}{l}\text { A woman, } 70-79 \text { years, admitted with pain related to breast cancer. During the admission, it became apparent that she } \\
\text { had metastases along with femur and vertebral fractures. A high dose of morphine (dose unknown) was necessary to } \\
\text { relieve her pain, which consequently resulted in a delirium. }\end{array}$ & $\begin{array}{l}1 \\
\text { (prescribing error) }\end{array}$ \\
\hline 28 & $\begin{array}{l}\text { A woman, } 80-89 \text { years, admitted due to a hip fracture and pain. For her restlessness and pain she was administered } \\
1 \mathrm{mg} \text { morphine, which probably caused a reduced level of consciousness. }\end{array}$ & $\begin{array}{l}1 \\
\text { (other error) }\end{array}$ \\
\hline
\end{tabular}

*Patients were categorised in age groups of 10 years to avoid traceability.

†Preventability was scored on a 6 -point Likert scale: $1=($ almost) no evidence of preventability; $2=$ small indications for preventability; $3=$ preventability not very likely, less than $50 \%$ but 'close call'; $4=$ preventability more than likely, more than $50 \%$ but 'close call'; $5=$ strong indications for preventability; $6=($ almost) certain indications of preventability.

fFor the judgement on preventability and type of error, the experts had access to all information on the electronic patient record and therefore to the whole context in which ADEs occurred. The types of error were prescribing error, administration error, other error (eg, side effects) or unknown.

ADEs, adverse drug events; COPD, chronic obstructive pulmonary disease.

In line with previous studies, ${ }^{7} 141517$ we found that dosing errors during prescribing and administering were the main cause of preventable ORADEs. Furthermore, $60 \%$ of the dosing errors in our study occurred in elderly patients ( $\geq 70$ years). In general, prescribing medication for elderly patients is challenging since polypharmacy, multimorbidity, and altered pharmacokinetics and pharmacodynamics of drugs are often present. Besides, this population will rapidly increase in the upcoming years. Specifically related to opioids, physicians also need to be aware of the higher sensitivity of elderly patients to the effects of opioids, ${ }^{35}$ and balancing between minimising the risk of addiction and side effects while effectively relieving pain. ${ }^{36} 37$ Taking into account all these factors while prescribing demands a lot from physicians during their busy daily hospital practice. A clinical decision support system (CDSS) can help physicians in this complex task by showing warnings and advice during prescribing, for example showing the most appropriate choice of medication for a given condition and/or by providing dosing recommendations. CDSS has shown to effectively reduce prescribing errors among hospitalised elderly patients ${ }^{38} 39$ and errors with medications of which the therapeutic effects are fast, such as opioids. ${ }^{40}$ Furthermore, a CDSS can also be effective in predicting which patients are at risk for ORADEs. Using retrospective data from gastrointestinal surgical patients, Minkowitz et $a l^{41}$ developed a risk-scoring model to identify patients with a high risk for experiencing an ORADE based on their clinical and demographic profiles. If developed specifically for elderly inpatients, such a prediction model could help physicians in determining the most appropriate and safe pain management strategy for these vulnerable patients. Finally, a CDSS could also be used to identify patients who might be suitable for pre-emptive genotyping, which involves metabolic testing prior to prescribing. ${ }^{42}$ Patients with high levels of pain despite using high doses of pain medication or patients who experience severe side effects while using common dosing schedules may especially benefit from such an intervention. ${ }^{43}$

Administering opioids is a task usually conducted by nurses. The dosing errors in our study were mostly related to injectable opioids. Error-prone activities, such as calculating the concentration and administration rate, ${ }^{14} 17$ require that nurses have sufficient arithmetic knowledge and follow the protocol for safe preparation and administration of injectable medication. However, in daily practice, some nurses have math anxiety, and on average arithmetic knowledge of nursing students seems moderate. ${ }^{44}$ Besides, nurse compliance with protocols for safe administration of injectable medication is considered low (around 20\%) ${ }^{46} 47$ and needs further attention. An intervention which might help to reduce dosing errors during opioid administration is the use of smart infusion pumps. These pumps have integrated medication libraries which allow nurses to 
Table 3 Characteristics of patients $(n=27)$ with ORADEs $(n=28)^{\star}$

\begin{tabular}{|lc|}
\hline Patient characteristics & \\
\hline Patients with an ADE, $\mathrm{n}$ & 27 \\
\hline Male sex, $\mathrm{n}(\%)$ & $11(41)$ \\
\hline Age, median years (IQR) & $76(66-83)$ \\
\hline Length of stay, median days (IQR) & $7(4-16)$ \\
\hline Non-elective admission, $\mathrm{n}(\%)$ & $19(70)$ \\
\hline Terminally ill prior to admission, $\mathrm{n}(\%)$ & $6(22)$ \\
\hline Total number of medical specialists, $\mathrm{n}(\%)$ & \\
\hline
\end{tabular}

\begin{tabular}{|cc|}
\hline 1 & $4(15)$ \\
\hline 2 & $2(7)$ \\
\hline 3 & $21(78)$ \\
\hline Primary specialisation during admission, $\mathrm{n}(\%)$ & \\
\hline Surgical & $7(26)$ \\
\hline Non-surgical & $20(74)$ \\
\hline Underwent invasive procedure, $\mathrm{n}(\%)$ & $9(33)$ \\
\hline Comorbidity†, $\mathrm{n}(\%)$ & \\
\hline No comorbidity & $0(0)$ \\
\hline Minor comorbidity & $3(11)$ \\
\hline Moderate comorbidity & $5(19)$ \\
\hline Significant comorbidity & $19(70)$ \\
\hline
\end{tabular}

*Presented on the patient level.

†The level of comorbidity was assessed by the experts after careful review of the information on patient records.

$A D E$, adverse drug event; ORADEs, opioid-related adverse drug events.

set the pump automatically to the right administration rate during administration. By doing so, the administration rate of smart pumps can be seen as a double-check of the nurses' own calculation. Smart pumps seem also effective in reducing programming errors. ${ }^{48}$ Furthermore, educational programmes for nurses about brand and generic names and pharmacology of opioids or side effects might increase their knowledge and awareness of risks related to dosing during the administration of opioids. $^{49-51}$

Overall, we think the ORADE frequency of $8 \%$ of all ADEs and $0.3 \%$ of all studied patient records found in our study is low and acceptable. However, although the frequency is low, the risk of serious consequences is high. Thus, new contributions to prevent ORADEs in future hospitalised patients need to be identified. Using the Safety-2 perspective may offer new opportunities to do so. ${ }^{52}$ In order to understand what happened when an adverse (drug) event occurred, it is also necessary to understand how work is done when the process goes well..$^{53}$ Since healthcare processes have become more complex nowadays, it may be helpful to visualise the current variable practice of prescribing and administering opioids from a multistakeholder perspective. ${ }^{54}$
Table 4 Clinical context of ORADEs $(n=28)^{\star}$

\begin{tabular}{|c|c|c|}
\hline Clinical context & $\begin{array}{l}\text { Non- } \\
\text { preventable } \\
\text { ADEs }(n=17)\end{array}$ & $\begin{array}{l}\text { Preventable } \\
\text { ADEs }(n=11)\end{array}$ \\
\hline \multicolumn{3}{|l|}{ Type of hospital, n (\%) } \\
\hline University, ADEs & $1(6)$ & $1(9)$ \\
\hline Tertiary teaching, ADEs & $6(35)$ & $4(36)$ \\
\hline General, ADEs & $10(59)$ & $6(55)$ \\
\hline $\begin{array}{l}\text { Weekend or national holiday } \\
\text { (yes), n (\%) }\end{array}$ & $5(31)$ & $2(18)$ \\
\hline \multicolumn{3}{|l|}{ Time, n (\%) } \\
\hline 08:00-17:00 & $6(35)$ & $5(45)$ \\
\hline 17:00-23:00 & $3(18)$ & $0(0)$ \\
\hline 23:00-08:00 & $2(12)$ & $3(27)$ \\
\hline Cannot be assessed & $6(35)$ & $3(27)$ \\
\hline \multicolumn{3}{|l|}{ Type of opioids (ATC code), $n$ (\%) } \\
\hline Opioid anaesthetics (N01AH03) & $2(12)$ & $1(9)$ \\
\hline $\begin{array}{l}\text { Natural opium alkaloids } \\
\text { (N02AA) }\end{array}$ & $9(53)$ & $8(73)$ \\
\hline $\begin{array}{l}\text { Natural opium alkaloids and } \\
\text { phenylpiperidine derivatives } \\
\text { (N02AA/N02AB, combination) }\end{array}$ & $1(6)$ & $1(9)$ \\
\hline $\begin{array}{l}\text { Phenylpiperidine derivatives } \\
\text { (N02AB) }\end{array}$ & $2(12)$ & $0(0)$ \\
\hline Other opioids (N02AX) & $1(6)$ & $0(0)$ \\
\hline $\begin{array}{l}\text { Drugs used in opioid } \\
\text { dependence (N07BC) }\end{array}$ & $2(12)$ & $1(9)$ \\
\hline \multicolumn{3}{|l|}{ Attributable factors $\ddagger, \mathrm{n}(\%)$} \\
\hline Technical & $0(0)$ & $0(0)$ \\
\hline Care-related & $3(19)$ & $8(80)$ \\
\hline Organisational & $2(13)$ & $4(40)$ \\
\hline Patient-related & $10(63)$ & $6(60)$ \\
\hline Violation & $0(0)$ & $1(10)$ \\
\hline Cannot be assessed & $3(19)$ & $1(10)$ \\
\hline Other & $1(6)$ & $0(0)$ \\
\hline
\end{tabular}

*Presented on the adverse event level.

†Preventability was scored on a 6-point Likert scale: $1=$ (almost) no evidence of preventability; $2=$ small indications for preventability; $3=$ preventability not very likely, less than $50 \%$ but 'close call';

$4=$ preventability more than likely, more than $50 \%$ but 'close call'; $5=$ strong indications for preventability; $6=$ (almost) certain indications of preventability. Not preventable ADEs were scored at 1-3; preventable ADEs were scored at 4-6.

$\ddagger$ These variables were missing for two patients: one in the preventable group and one in the non-preventable group. Moreover, it was possible to select more than one option for this question. ADE, adverse drug event; ATC, Anatomical Therapeutic Chemical; ORADE, opioid-related adverse drug event.

\section{Strengths and limitations}

Opioids are in the top 10 of drug types that cause fatal medication errors. ${ }^{8}$ Hence, focusing on the detailed description of the nature of ORADEs was important and necessary. Another strength of this study is that it was based on a comprehensive ADE detection method and conducted in a broad sample of all hospital admissions. Most previous studies, which described the nature 
of ORADEs, are based on medication-related incident reports. Furthermore, data were gathered over an extended period of time within a randomly selected sample of one-third of all Dutch hospitals.

This study also has some limitations. First, in all three AE studies, the population consisted of relatively many older and deceased patients. Therefore, it is not possible to generalise the results to all Dutch hospital population. To make the study sample more representative for the Dutch hospital population, weighting the results (ie, correcting for type of hospital, study period and discharge status) would be a solution which has been used in previous studies of our research group. However, since the total amount of ORADEs was low, we chose not to weight our results as this had little effect and makes interpretation difficult. Second, overall agreement frequencies between physicians were moderate. This could have led to different assessments or different scores if other experts were involved. This should be taken into account when interpreting our results. However, a previous review of studies focusing on assessing AEs showed also moderate to substantial inter-rater reliability ${ }^{55}$ For this reason, patient records in all Dutch $\mathrm{AE}$ studies have been assessed by the same experts as much as possible, and over the years these experts have not become stricter or lenient in their judgement of AEs and their preventability. ${ }^{56}$ Third, due to this low number of ORADEs, it was not possible to compare the events over the three study periods. Therefore, we cannot conclude whether the low number is a positive finding and if the occurrence of ORADEs increased or decreased over time. Fourth, our post-hoc analysis was based on the information previously recorded by the experts in an $\mathrm{AE}$ database and on the assessment conducted by these physicians. Therefore, some information could be missing, and interpreting the assessment of preventability was difficult for us in one case, resulting in a non-preventable ORADE. Furthermore, this was also the reason that the harm could not be further categorised according to the National Coordinating Council for Medication Error Reporting and Prevention (NCCMERP) Index for Categorizing Medication Errors. ${ }^{57}$ Besides, the retrospective interpretation can also be biased by temporal views. The current opinion is that prescribing opioids should be minimised due to the harm of opioids, which is supported by updated guidelines. ${ }^{58}$ This view changed throughout the years and may not have been recognised 15 years ago, when the focus was mainly on alleviating suffering of pain. This change in opinion may have increased alertness when prescribing or administering opioids, which could have led to less ORADEs. However, our study showed that ORADEs still occur and publishing about them could serve as a method of increasing awareness.

\section{CONCLUSION}

Only $8 \%$ of ADEs identified in our sample were related to opioids, $0.3 \%$ of all studied patient records. Although the frequency is low, the risk of serious consequences is high. We recommend to use our findings to increase awareness among physicians and nurses. Future interventions should focus on safe dosing of opioids when prescribing and administering, especially in elderly patients.

Acknowledgements We would like to thank all participating hospitals and independent nurses and physicians for their cooperation during the data collection on the three Dutch adverse event studies. We also thank Peter Spreeuwenberg, MSc, for double-checking the statistical analyses, and Catherine Combee-Duffy, MANP, for improving the readability of this article.

Contributors BCFMS, MM, JEK, ML, MdB and CW designed the study and developed the study protocol. BCFMS and MM organised the selection and classification of ORADES. JEK and IJ double-checked this classification. BCFMS and MM performed statistical analyses and interpreted the analytical results. BCFMS, JEK and IJ wrote the manuscript. MdB and CW supervised the study. All authors made critical revisions and approved the final version of the manuscript.

Funding This study was funded by the Dutch Ministry of Health, Welfare and Sport, with project title Monitor Zorggerelateerde Schade 2015-2018.

Competing interests None declared.

Patient and public involvement Patients and/or the public were not involved in the design, or conduct, or reporting, or dissemination plans of this research.

Patient consent for publication Not required.

Ethics approval The medical ethical committee of the Amsterdam UMC, Vrije Universiteit Amsterdam waived the requirement of informed consent (protocol numbers: $2005.146,2009.130,2016.282$ ) as they found the scope of the study outside the Dutch Medical Research (Human Subjects) Act.

Provenance and peer review Not commissioned; externally peer reviewed.

Data availability statement No data are available. All data relevant to the study are included in the article or uploaded as supplementary information.

Open access This is an open access article distributed in accordance with the Creative Commons Attribution Non Commercial (CC BY-NC 4.0) license, which permits others to distribute, remix, adapt, build upon this work non-commercially, and license their derivative works on different terms, provided the original work is properly cited, appropriate credit is given, any changes made indicated, and the use is non-commercial. See: http://creativecommons.org/licenses/by-nc/4.0/.

\section{ORCID iDs}

Bernadette Clara Francisca Maria Schutijser http://orcid.org/0000-0002-8957-4590 Joanna Ewa Klopotowska http://orcid.org/0000-0002-9707-5740

Marco Moesker http://orcid.org/0000-0002-3546-760X

Martine de Bruijne http://orcid.org/0000-0003-1838-1158

\section{REFERENCES}

1 Schepens MHJ, Leusink M, de Vries SE, et al. [Increase in opioid prescribing in extramural care in the Netherlands: assessment of use and prescription behaviour, based on claims data]. Ned Tijdschr Geneeskd 2019;163:D3854.

2 Lyden J, Binswanger IA. The United States opioid epidemic. Semin Perinatol 2019;43:123-31.

3 AMA. Physicians' progress to reverse the nation's opioid epidemic: American Medical Association, 2018. Available: https://www.amaassn.org/sites/default/files/media-browser/public/physicians/patientcare/opioid-task-force-progress-report.pdf [Accessed 8 Aug 2018].

4 Overheid.nl. Geneesmiddelenbeleid. Den Haag, the Netherlands: Overheid.nl, 2019. Available: https://zoek.officielebekendmakingen. nl/kst-29477-537.html [Accessed 19 Mar 2019].

5 Laatikainen O, Miettunen J, Sneck S, et al. The prevalence of medication-related adverse events in inpatients-a systematic review and meta-analysis. Eur J Clin Pharmacol 2017;73:1539-49.

6 Mihajlovic S, Gauthier J, MacDonald E. Patient characteristics associated with adverse drug events in hospital: an overview of reviews. Can J Hosp Pharm 2016;69:294-300.

7 Mc Donnell C. Opioid medication errors in pediatric practice: four years' experience of voluntary safety reporting. Pain Res Manag 2011;16:93-8.

8 Saedder EA, Brock B, Nielsen LP, et al. Identifying high-risk medication: a systematic literature review. Eur J Clin Pharmacol 2014;70:637-45. 
9 Kane-Gill SL, Rubin EC, Smithburger PL, et al. The cost of opioidrelated adverse drug events. J Pain Palliat Care Pharmacother 2014;28:282-93.

10 Oderda GM, Gan TJ, Johnson BH, et al. Effect of opioid-related adverse events on outcomes in selected surgical patients. J Pain Palliat Care Pharmacother 2013;27:62-70.

11 Kessler ER, Shah M, Gruschkus SK, et al. Cost and quality implications of opioid-based postsurgical pain control using administrative claims data from a large health system: opioid-related adverse events and their impact on clinical and economic outcomes. Pharmacotherapy 2013;33:383-91.

12 de Vries EN, Ramrattan MA, Smorenburg SM, et al. The incidence and nature of in-hospital adverse events: a systematic review. Qual Saf Health Care 2008;17:216-23.

13 Bates DW, Cullen DJ, Laird N, et al. Incidence of adverse drug events and potential adverse drug events. Implications for prevention. ade prevention Study Group. JAMA 1995;274:29-34.

14 Doherty C, Mc Donnell C. Tenfold medication errors: 5 years experience at a university-affiliated pediatric hospital. Pediatrics 2012:129:916-24.

15 Heneka N, Shaw T, Rowett D, et al. Opioid errors in inpatient palliative care services: a retrospective review. BMJ Support Palliat Care 2018;8:175-9.

16 Shafi S, Collinsworth AW, Copeland LA, et al. Association of opioidrelated adverse drug events with clinical and cost outcomes among surgical patients in a large integrated health care delivery system. JAMA Surg 2018;153:757-63.

17 Dy SM, Shore AD, Hicks RW, et al. Medication errors with opioids: results from a national reporting system. $J$ Opioid Manag 2007;3:189-94.

18 Cousins DH, Gerrett D, Warner B. A review of medication incidents reported to the National reporting and learning system in England and Wales over 6 years (2005-2010). Br J Clin Pharmacol 2012;74:597-604.

19 Noble DJ, Pronovost PJ. Underreporting of patient safety incidents reduces health care's ability to quantify and accurately measure harm reduction. J Patient Saf 2010;6:247-50.

20 Yung H-P, Yu S, Chu C, et al. Nurses' attitudes and perceived barriers to the reporting of medication administration errors. J Nurs Manag 2016;24:580-8

21 Baker GR, Norton PG, Flintoft V, et al. The Canadian adverse events study: the incidence of adverse events among hospital patients in Canada. CMAJ 2004;170:1678-86.

22 Brennan TA, Leape LL, Laird NM, et al. Incidence of adverse events and negligence in hospitalized patients. Results of the Harvard medical practice study I. N Engl J Med 1991;324:370-6.

23 Baines RJ, Langelaan M, de Bruijne MC, et al. Changes in adverse event rates in hospitals over time: a longitudinal retrospective patient record review study. BMJ Qual Saf 2013;22:290-8.

24 Langelaan M, De Bruijne MC, Baines RJ, et al. Dutch adverse event study 2011/2012 Utrecht: NIVEL/EMGO+, 2013. Available: https:// www.nivel.nl/sites/default/files/bestanden/monitor_zorggerelateerde schade_2011_2012.pdf [Accessed 12 Dec 2018]

25 Baines RJ, Langelaan M, de Bruijne MC, et al. Is researching adverse events in hospital deaths a good way to describe patient safety in hospitals: a retrospective patient record review study. BMJ Open 2015;5:e007380.

26 Vincent C, Neale G, Woloshynowych M. Adverse events in British hospitals: preliminary retrospective record review. BMJ 2001;322:517-9.

27 Zegers M, de Bruijne MC, Wagner C, et al. Adverse events and potentially preventable deaths in Dutch hospitals: results of a retrospective patient record review study. Qual Saf Health Care 2009;18:297-302.

28 Damen NL, Baines R, Wagner C, et al. Medication-Related adverse events during hospitalization: a retrospective patient record review study in the Netherlands. Pharmacoepidemiol Drug Saf 2017;26:32-9.

29 Baines R, Langelaan M, de Bruijne $M$, et al. How effective are patient safety initiatives? A retrospective patient record review study of changes to patient safety over time. BMJ Qual Saf 2015;24:561-71.

30 van Vuren W, Shea CE, van der Schaaf TW. The development of an incident analysis tool for the medical field. Eindhoven: Eindhoven University of Technology, 1997.

31 Morimoto T, Sakuma M, Matsui K, et al. Incidence of adverse drug events and medication errors in Japan: the JADE study. J Gen Intern Med 2011;26:148-53.

32 WHO. Medication errors: technical series on safer primary care. Geneva: World Health Organization, 2016. http://apps.who.int/ iris/bitstream/handle/10665/252274/9789241511643-eng.pdf? sequence $=1$
33 WHO. ATC/DDD index, 2017. Available: https://www.whocc.no/atc ddd index/?code=N02A [Accessed 10 Jul 2018]

34 de Vet HCW, Mokkink LB, Terwee CB, et al. Clinicians are right not to like Cohen's к. BMJ 2013;346:f2125.

35 Huang AR, Mallet L. Prescribing opioids in older people. Maturitas 2013;74:123-9.

36 Aronson JK. Balanced prescribing - principles and challenges. $\mathrm{Br} \mathrm{J}$ Clin Pharmacol 2012;74:566-72.

37 Wallwork RS, Chipidza FE, Stern TA. Obstacles to the prescription and use of opioids. Prim Care Companion CNS Disord 2016;18.

38 Scott IA, Pillans PI, Barras M, et al. Using EMR-enabled computerized decision support systems to reduce prescribing of potentially inappropriate medications: a narrative review. Ther $\mathrm{Adv}$ Drug Saf 2018;9:559-73.

39 Clyne B, Bradley MC, Hughes C, et al. Electronic prescribing and other forms of technology to reduce inappropriate medication use and polypharmacy in older people: a review of current evidence. Clin Geriatr Med 2012;28:301-22.

40 Durieux P, Trinquart L, Colombet I, et al. Computerized advice on drug dosage to improve prescribing practice. Cochrane Database Syst Rev 2008;3:CD002894.

41 Minkowitz HS, Scranton R, Gruschkus SK, et al. Development and validation of a risk score to identify patients at high risk for opioid-related adverse drug events. J Manag Care Spec Pharm 2014;20:948-58.

42 Hinderer M, Boeker M, Wagner SA, et al. Integrating clinical decision support systems for pharmacogenomic testing into clinical routine - a scoping review of designs of user-system interactions in recent system development. BMC Med Inform Decis Mak 2017:17:81.

43 Agarwal D, Udoji MA, Trescot A. Genetic testing for opioid pain management: a primer. Pain Ther 2017;6:93-105.

44 Simonsen BO, Daehlin GK, Johansson I, et al. Differences in medication knowledge and risk of errors between graduating nursing students and working registered nurses: comparative study. BMC Health Serv Res 2014;14:580.

45 Williams B, Davis S. Maths anxiety and medication dosage calculation errors: a scoping review. Nurse Educ Pract 2016;20:139-46.

46 Schilp J, Boot S, de Blok C, et al. Protocol compliance of administering parenteral medication in Dutch hospitals: an evaluation and cost estimation of the implementation. BMJ Open 2014;4:e005232.

47 Schutijser B, Klopotowska JE, Jongerden I, et al. Nurse compliance with a protocol for safe injectable medication administration: comparison of two multicentre observational studies. BMJ Open 2018;8:e019648.

48 Ohashi K, Dalleur O, Dykes PC, et al. Benefits and risks of using smart pumps to reduce medication error rates: a systematic review. Drug Saf 2014;37:1011-20.

49 Murnion BP, Gnjidic D, Hilmer SN. Prescription and administration of opioids to hospital in-patients, and barriers to effective use. Pain Med 2010;11:58-66.

50 Jho HJ, Kim Y, Kong KA, et al. Knowledge, practices, and perceived barriers regarding cancer pain management among physicians and nurses in Korea: a nationwide multicenter survey. PLOS One 2014;9:e105900.

51 AlReshidi N, Long T, Darvill A. A systematic review of the impact of educational programs on factors that affect nurses' postoperative pain management for children. Compr Child Adolesc Nurs 2018;41:9-24.

52 Furniss D, Lyons I, Franklin BD, et al. Procedural and documentation variations in intravenous infusion administration: a mixed methods study of policy and practice across 16 Hospital trusts in England. BMC Health Serv Res 2018:18:270.

53 Hollnagel E, Wears R, Braithwaite J. From safety-I to safety-II: a white paper. The resilient healthcare net: published simultaneously by the University of southern Denmark, University of Florida, USA, and Australia. Macquarie University, 2015.

54 Clay-Williams R, Hounsgaard J, Hollnagel E. Where the rubber meets the road: using FRAM to align work-as-imagined with work-as-done when implementing clinical guidelines. Implement Sci 2015;10:125.

55 Hanskamp-Sebregts M, Zegers M, Vincent C, et al. Measurement of patient safety: a systematic review of the reliability and validity of adverse event detection with record review. BMJ Open 2016;6:e011078.

56 Baines RJ. Intra-rater agreement in adverse event studies: stability of assessment of adverse events over time, 2018. Available: https:// www.nivel.nl/sites/default/files/bestanden/Proefschrif_Rebecca Baines_Monitoring_adverse_events_in_hospitals.pdf [Accessed 26 Jun 2020]. 
57 Hartwig SC, Denger SD, Schneider PJ. Severity-indexed, incident report-based medication error-reporting program. Am J Hosp Pharm 1991;48:2611-6.
58 Verenso. Richtlijn Pijn.Herkenning en behandeling van pijn bij kwetsbare ouderen. Utrecht, 2016. Available: https://www.verenso. nl/_asset/_public/Richtlijnen_kwaliteit/richtlijnen/database/VER-00332-Richtlijn-Pijn-deel2-v5LR.pdf [Accessed 20 Jun 2020]. 